Proyecciones

Vol. 14, No 2, pp. 83-88, December 1995

Universidad Católica del Norte

Antofagasta - Chile

\title{
GLOBAL SMOOTHNESS PRESERVATION BY SINGULAR INTEGRALS
}

\author{
George A. Anastassiou \\ Memphis State University, U. S. A.
}

\begin{abstract}
Here is established that the well-known singular integrals of Picard, Poisson-Cauchy and Gauss-Weierstrass fulfill the "global smoothness preservation" property. I.e., they "ripple" less than the function they are applied on, that is producing a nice approximation to the unit. The associated inequalities are sharp.
\end{abstract}

AMS 1991 Mathematics Subject Classification:

Primary: 26A15, 41A17, $41 A 35$.

Secondary: $26015,41 A 44$.

Key Words and Phrases: Best constant, global smoothness preservation, singular integral, sharp inequality. 


\section{Introduction}

Let $f$ be a function from $\mathbf{R}$ into itself. We call

$$
\omega_{1}(f, h):=\sup _{\substack{x, y \\|x-y| \leq h}}|f(x)-f(y)|, \quad h>0
$$

the first modulus of continuity of $f$. Here we consider $f$ such that $\omega_{1}(f, h)<+\infty$, for any $h>0$.

For $\zeta>0$ with $\zeta \rightarrow 0$, we consider the well-known Picard, PoissonCauchy and Gauss-Weierstrass singular integrals

$$
\begin{gathered}
P_{\zeta}(f, x):=\frac{1}{2 \zeta} \cdot \int_{-\infty}^{\infty} f(x+t) \cdot e^{-|t| / \zeta} \cdot d t, \\
Q_{\zeta}(f, x):=\frac{\zeta}{\pi} \cdot \int_{-\pi}^{\pi}\left(\frac{f(x+t)}{t^{2}+\zeta^{2}}\right) \cdot d t
\end{gathered}
$$

and

$$
W_{\zeta}(f, x):=\frac{1}{\sqrt{\pi \cdot \zeta}} \cdot \int_{-\pi}^{\pi} f(x+t) \cdot e^{-t^{2} / \zeta} \cdot d t .
$$

The above integrals are positive linear operators with respect to $f$. Furthermore, here we consider only $f$ such that $P_{\zeta}(f, x), Q_{\zeta}(f, x), W_{\zeta}(f, x) \in$ $\mathbf{R}$, for all $x \in \mathbf{R}$. In particular, notice that (for $i d(x)=x$ )

$$
\begin{gathered}
P_{\zeta}(i d, x)=x \in \mathrm{R}, \\
Q_{\zeta}(i d, x)=\frac{2 x}{\pi} \cdot \tan ^{-1} \frac{\pi}{\zeta} \in \mathrm{R},
\end{gathered}
$$

and

$$
W_{\zeta}(i d, x)=x \cdot \operatorname{Erf}\left(\frac{\pi}{\sqrt{\zeta}}\right) \in \mathbf{R}, \text { all } x \in \mathbf{R} .
$$

To get (1.5) we use that

$$
\frac{1}{2 \zeta} \cdot \int_{-\infty}^{\infty} e^{-|t| / \zeta} d t=1
$$

to get (1.6) we use that

$$
\frac{\zeta}{\pi} \cdot \int_{-\pi}^{\pi} \frac{d t}{t^{2}+\zeta^{2}}=\frac{2}{\pi} \cdot \tan ^{-1} \frac{\pi}{\zeta},
$$


finally, to get (1.7) we use that

$$
\operatorname{Erf}(x):=\frac{2}{\sqrt{\pi}} \cdot \int_{0}^{x} e^{-t^{2}} \cdot d t .
$$

Obviously, id is a function from $\mathbf{R} \rightarrow \mathbf{R}$ such that

$$
\omega_{1}(i d, h)=h<+\infty \text {, any } h>0 .
$$

The above operators (1.2), (1.3), (1.4) have been studied extensively in [2]. In [2] the authors obtain the degree of convergence of these operators to the unit with rates over the class of Hölder-continuous functions.

In [1] the author obtains some more refined results, in the same direction as in [2], however, only over the set of $\left(C_{2 \pi}\right) 2 \pi$-periodic continuous functions on $R$, where a monotonicity assumption from [2] regarding the modulus of continuity there is dropped.

In this note the author establishes that the operators (1.2), (1.3), (1.4) when applied to $f$ do not "ripple" more than $f$. I.e., operators (1.2), (1.3), (1.4) fulfill the "global smoothness preservation" property, and the associated inequalities are sharp, and more precisely are attained. The above "smoothness preservation" property indicates that the operators (1.2), (1.3), (1.4) approximate nicely the unit operator.

\section{Main Results}

Theorem 1. Let the function $f: R \rightarrow R$ with $\omega_{1}(f, h)<+\infty$, for any $h>0$, such that $P_{\zeta}(f, x), Q_{\zeta}(f, x), W_{\zeta}(f, x) \in R$, for all $x \in R$. Here take $\zeta>0$ and $\zeta \rightarrow 0$. Then

$$
\omega_{1}\left(Q_{\zeta}(f), h\right) \leq \frac{2}{\pi} \cdot \tan ^{-1} \frac{\pi}{\zeta} \cdot \omega_{1}(f, h)
$$

and

$$
\omega_{1}\left(W_{\zeta}(f), h\right) \leq \operatorname{Erf}\left(\frac{\pi}{\sqrt{\zeta}}\right) \cdot \omega_{1}(f, h),
$$

for any $h>0$. 


\section{Proof.}

Inequality (2.1): Note that

$$
P_{\zeta}(f, x)-P_{\zeta}(f, y)=\frac{1}{2 \zeta} \cdot \int_{-\infty}^{\infty}(f(x+t)-f(y+t)) \cdot e^{-|t| / \zeta} \cdot d t
$$

Thus

$$
\begin{aligned}
\left|P_{\zeta}(f, x)-P_{\zeta}(f, y)\right| & \leq \frac{1}{2 \zeta} \cdot \int_{-\infty}^{\infty}|f(x+t)-f(y+t)| \cdot e^{-|t| / \zeta} \cdot d t \\
& \leq \frac{1}{2 \zeta} \cdot \int_{-\infty}^{\infty} \omega_{1}(f,|x-y|) \cdot e^{-|t| / \zeta} \cdot d t \\
& =\omega_{1}(f,|x-y|), \text { by }(1.8) .
\end{aligned}
$$

Now (2.1) is obvious.

Inequality (2.2): See that

$$
Q_{\zeta}(f, x)-Q_{\zeta}(f, y)=\frac{\zeta}{\pi} \cdot \int_{-\pi}^{\pi} \frac{(f(x+t)-f(y+t))}{t^{2}+\zeta^{2}} \cdot d t
$$

Hence

$$
\begin{aligned}
\left|Q_{\zeta}(f, x)-Q_{\zeta}(f, y)\right| & \leq \frac{\zeta}{\pi} \cdot \int_{-\pi}^{\pi} \frac{|f(x+t)-f(y+t)|}{t^{2}+\zeta^{2}} \cdot d t \\
& \leq \frac{\zeta}{\pi} \cdot \int_{-\pi}^{\pi} \frac{\omega_{1}(f,|x-y|)}{t^{2}+\zeta^{2}} \cdot d t \\
& =\omega_{1}(f,|x-y|) \cdot \frac{2}{\pi} \cdot \tan ^{-1} \frac{\pi}{\zeta}, \text { by }(1.9)
\end{aligned}
$$

The validity of (2.2) now is clear.

Inequality (2.3): Observe that

$$
W_{\zeta}(f, x)-W_{\zeta}(f, y)=\frac{1}{\sqrt{\pi \zeta}} \cdot \int_{-\pi}^{\pi}(f(x+t)-f(y+t)) \cdot e^{-t^{2} / \zeta} \cdot d t
$$

Therefore

$$
\begin{aligned}
\left|W_{\zeta}(f, x)-W_{\zeta}(f, y)\right| & \leq \frac{1}{\sqrt{\pi \zeta}} \cdot \int_{-\pi}^{\pi}|f(x+t)-f(y+t)| \cdot e^{-t^{2} / \zeta} \cdot d t \\
& \leq \frac{1}{\sqrt{\pi \zeta}} \cdot \omega_{1}(f,|x-y|) \cdot \int_{-\pi}^{\pi} e^{-t^{2} / \zeta} \cdot d t \\
& =\operatorname{Erf}\left(\frac{\pi}{\sqrt{\zeta}}\right) \cdot \omega_{1}(f,|x-y|), \text { from }(1.10) .
\end{aligned}
$$


I.e.,

$$
\left|W_{\zeta}(f, x)-W_{\zeta}(f, y)\right| \leq \operatorname{Er} f\left(\frac{\pi}{\sqrt{\zeta}}\right) \cdot \omega_{1}(f,|x-y|), \text { all } x, y \in \mathbf{R} .
$$

The last establishes (2.3).

Optimality is obtained in

Theorem 2. Inequalities (2.1), (2.2), (2.3) are sharp, in fact they are attained by the identity function.

Proof. Note that the $i d$-function belongs to the class of functions under consideration, for this see the related discussion in $\$ 1$ and $(1.5),(1.6),(1.7)$.

Inequality (2.1): See that

$$
\begin{aligned}
P_{\zeta}(i d, x)-P_{\zeta}(i d, y) & =\frac{1}{2 \zeta} \cdot \int_{-\infty}^{\infty}((x+t)-(y+t)) \cdot e^{-|t| / \zeta} \cdot d t \\
& =(x-y) \cdot \frac{1}{2 \zeta} \cdot \int_{-\infty}^{\infty} e^{-|t| / \zeta} \cdot d t \\
& =x-y, \text { by }(1.8) .
\end{aligned}
$$

That is

$$
\omega_{1}\left(P_{\zeta}(i d), h\right)=\omega_{1}(i d, h)
$$

any $h>0$.

Inequality (2.2): Observe that

$$
\begin{aligned}
Q_{\zeta}(i d, x)-Q_{\zeta}(i d, y) & =(x-y) \cdot \frac{\zeta}{\pi} \cdot \int_{-\pi}^{\pi} \frac{d t}{t^{2}+\zeta^{2}} \\
& =(x-y) \cdot \frac{2}{\pi} \cdot \tan ^{-1} \frac{\pi}{\zeta}, \text { by }(1.9) .
\end{aligned}
$$

I.e.,

$$
\omega_{1}\left(Q_{\zeta}(i d), h\right)=\omega_{1}(i d, h) \cdot \frac{2}{\pi} \cdot \tan ^{-1} \frac{\pi}{\zeta}
$$

any $h>0$. 
Inequality (2.3): Similarly we have that

$$
\begin{aligned}
W_{\zeta}(i d, x)-W_{\zeta}(i d, y) & =(x-y) \cdot \frac{1}{\sqrt{\pi \zeta}} \cdot \int_{-\pi}^{\pi} e^{-t^{2} / \zeta} \cdot d t \\
& =(x-y) \cdot \operatorname{Erf}\left(\frac{\pi}{\sqrt{\zeta}}\right), \text { by }(1.10) .
\end{aligned}
$$

That is,

$$
\omega_{1}\left(W_{\zeta}(i d), h\right)=\omega_{1}(i d, h) \cdot \operatorname{Erf}\left(\frac{\pi}{\sqrt{\zeta}}\right)
$$

for any $h>0$.

Q.E.D.

Remark 1. As $\zeta \rightarrow 0$ note that both $\left(\frac{2}{\pi} \cdot \tan ^{-1} \frac{\pi}{\zeta}\right)$ and $\operatorname{Erf}\left(\frac{\pi}{\sqrt{\zeta}}\right)$ tend to 1 , showing that the (R.H.S.)'s of $(2.1),(2.2),(2.3)$ for very small $\zeta$ are about the same.

\section{References}

[1] S.G. Gal, "Remark on the Degree of Approximation of Continuous Functions by Singular Integrals," Math. Nachr. 164, pp. 197 199 (1993).

[2] R.N. Mohapatra and R.S. Rodriguez, "On the Rate of Convergence of Singular Integrals for Hölder Continuous Functions," Math. Nachr. 149, pp. $117-124$ (1990).

Received : April 10, 1995.

George A. Anastassiou

Department of Mathematical Sciences

Memphis State University

Memphis, Tennessee 38152

U. S. A. 